\title{
ARQUIVO ELETRÔNICO: UMA BOMBA RELÓGIO NO MUNDO DA PROVA DIGITAL?
}

\section{ELETRONIC ARCHIVE: A TIME BOMB IN THE WORLD OF DIGITAL EVIDENCE?}

\begin{abstract}
FRANCISCO PACHECO DE ANDRADE
Professor Auxiliar da Escola de Direito da Universidade do Minho (Portugal). Director do Curso de Mestrado em Direito e Informática da Escola de Direito da Universidade do Minho. Doutoramento em Direito, Ciências Jurídico-Privatísticas (Universidade do Minho - 2009 ). MSc in Electronic Information Management ( University of Sheffield - 1996) com equivalência ao Grau de Mestre em Informática de Gestão ( Universidade do Minho 1997). Pós-Graduado em Direito da Comunicação - Faculdade de Direito da Universidade de Coimbra 1993. franc.andrade.direito@gmail.com
\end{abstract}

\begin{abstract}
RESUMO
A crescente utilização de documentos eletrônicos e o acelerado desenvolvimento de tecnologias, programas e formatos digitais colocam questões urgentes relativamente à necessidade de assegurar a preservação dos documentos desmaterializados e a sua compatibilização com os requisitos de utilização das assinaturas eletrônicas. Várias abordagens têm sido equacionadas, desde a emulação ou preservação de programas (software) e equipamentos (hardware) até à migração e à utilização de sofisticados recursos tecnológicos que permitem a autenticação e a datação dos documentos. De grande relevo para o direito probatório se revestirá ainda a existência de prestadores de serviços de confiança, incluindo prestadores de serviços de arquivo de longo termo de documentos eletrônicos.
\end{abstract}

Palavras-chave: Arquivo Eletrônico; Assinatura Eletrônica; Prova Digital; Emulação; Migração; Terceiros de Confiança.

\begin{abstract}
The increasing use of electronic documents and the accelerated development of technologies, programs and digital formats pose urgent questions as to the need to ensure the preservation of dematerialized documents and their compliance with the requirements for use of electronic signatures. Various approaches have been envisaged, since the emulation or preservation programs (software) and equipment (hardware) to migration and the use of sophisticated technological resources that enable authentication and dating of documents. Of great importance for the evidential law is still take the existence of trust service providers, including long-term electronic document filing service providers.
\end{abstract}

Keywords: Digital Evidence; Eletronic File; Eletronic Signature; Emulation; Migration; Relying Parties. 


\section{SUMÁRIO \\ INTRODUÇAO; 1 EMULAÇÃO; 2 MIGRAÇÃO; 3 OUTROS PONTOS A CONSIDERAR; CONCLUSÃO; REFERÊNCIAS.}

\section{INTRODUÇÃO}

Uma das maiores dificuldades na utilização dos documentos electrónicos como meio de prova prende-se com a constante e célere evolução tanto dos programas de software como do hardware necessário à sua leitura ${ }^{1}$. Esta rápida e constante evolução poderá até rapidamente tornar obsoletos programas e equipamentos necessários à leitura de documentos arquivados ${ }^{2}$. Assim, torna-se premente encarar estratégias eficientes que permitam não só o arquivo de documentos electrónicos, mas também a sua ulterior leitura, em qualquer momento em que tal seja necessário ${ }^{3}$. E há que assegurar uma conservação dos documentos, independentemente das futuras evoluções tecnológicas e de software, em termos compatíveis com a necessária comprovação da identificação do autor do documento e da integridade do escrito electrónico, de modo compatível com a utilização das assinaturas electrónicas digitais. Esta conservação ${ }^{4}$

\footnotetext{
1 - Cfr. Sofie Van den Eynde, "Digitale Archivering: een juridische stand van zaken vanuit Belgisch perspectief. Deel 1" (Arquivamento Digital: a situação jurídica atual a partir da perspective belga. Parte 1), Digitale Archivering in Vlaamse Instellingen en Diensten - DAVID, Faculteit Rechtsgeleerheid, Interdisciplinair Centrum voor Recht en Informatica, Leuven 2001, pág. 26: "De snelle evolutie in tekstverwerkingssoftware maakt dat de bestanden na verloop van tijd niet meer lesbaar zullen zijn. Bijgevolg zullen er strikte eisen moeten gesteld worden aan het format van het bestand en aan de dragger waarop het bestand wordt opgeslagen" (A rápida evolução no software de processamento de texto faz com que documentos, passado algum tempo, já não sejam legíveis. Por isso, devem ser impostas normas exactas relativas ao formato de um documento e ao seu suporte).

2 - "Computer hardware and software are undergoing constant and rapid changes and nobody can foresee how electronic information will be processed twenty or thirty years from now. ", cfr. Jos Dumortier, "EGovernment and Digital Preservation”, http://www.unizar.es/derecho/fyd/lefis/documentos/Albareccin_JosDumortier.pdf pág. 1.

3 - "How can we guarantee that the electronic documents that are being stored today will still be readable by the computers and programs that will be used in the future?", cfr. Jos Dumortier, "E-Government and Digital Preservation", citada, pag 1. Cfr. ainda Jos Dumortier e Sofie Van den Eynde;"Electronic Signatures

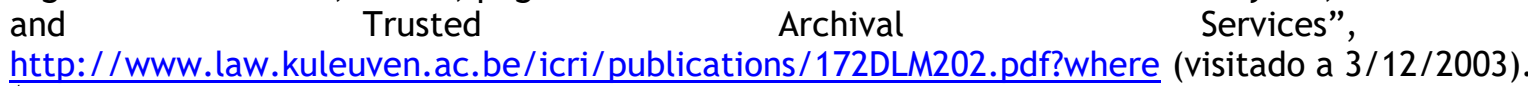

4 - Esta necessidade de conservação terá também que ter em atenção o problema da própria durabilidade dos suportes. Cfr. Sofie Van den Eynde, "Digitale Archivering: een juridische stand van zaken vanuit Belgische perspectief. Deel 1", citado, pág. 82, ponto A - "Het aspect"Tijd" en digitale duurzaamheid" ( 0 aspecto do "tempo" e da durabilidade digital): "Vooreerst is er het problem van de duurzaamheid van de drager. In vergelijking met papier is de levensduur van een digitale drager uiterst kort. Diskettes gaan
} 
reveste-se da maior importância para o comércio electrónico sob pena de se colocar em risco a própria força probatória dos documentos electrónicos ${ }^{5}$.

A questão da conservação dos documentos electrónicos tem preocupado sobretudo os arquivistas e informáticos ${ }^{6}$. Infelizmente, os juristas não se têm dedicado muito a este tema, o qual, no entanto, se reveste de enorme importância no plano jurídico, sobretudo no plano das prova dos contratos e transações. A existência de arquivos electrónicos e de condições para uma efetiva leitura, durante longos intervalos de tempo, dos documentos electrónicos arquivados, poderá tornar-se decisiva para que a opção pelo comércio electrónico possa ser realmente assumida em condições que permitam um elevado nível de segurança das transações e de confiança por parte dos agentes económicos. É que a conservação dos documentos electrónicos ${ }^{7}$ torna-se essencial tanto para que possa ser produzida prova de atos jurídicos em Tribunal, em caso de contencioso, como para que os próprios particulares possam comprovar determinados atos perante terceiros ou perante o Estado ${ }^{8}$. E essa conservação haverá de ser assegurada por períodos suficientemente longos que possibilitem, nomeadamente, a leitura dos documentos a

hoogstens enkele jaren mee, CD-roms ten hoogste enkele tientallen jaren, alvorens er informatieverlies optreedt" (Primeiro existe o problema da durabilidade do suporte. Em comparação com o papel, a duração de um suporte digital é extremamente curta. As disquetes duram no máximo alguns anos e os CDRoms algumas dezenas de anos, antes de ocorrerem perdas de informação). Esta questão terá que colocar-se hoje em termos diferenciados, devido à emergência da computação distribuída em rede ou "cloud computing", mas a necessidade de uma consideração pelo direito das matérias relativas à utilização de diferentes formatos e suportes mantém-se.

5 - "En outre, de même que pour l'intelligibilité, il est nécessaire d'entendre l'archivage électronique comme une conservation "active" à même de garantir la restitution de l'identification de l'auteur et de l'intégrité de l'écrit électronique. A défaut d'une adaptation des moyens d'archivage électronique aux évolutions technologiques, la sécurité et la durabilité (au sens "détection de toute altération ou modification ultérieure de l'acte") exigées de la conservation électronique pour garantir la force probante de l'écrit électronique risqueraient d'être amoindries", cfr. Eric Caprioli e Gerard Weisz, "Archivage électronique: des contraintes juridiques et technologiques“, Journal du Net, 2004.

6 -.- para uma abordagem arquivística e informática sobre este tema cfr. Aleksej Jerman Blazic "Long Term Trusted Archive Services", in Digital Society 2007, ICDS '07 - First International Conference on the Digital Society, IEEE 2007.

7 .- Cfr. Aleksej Blazic, citado: "The original environment where digital data is crreated may change or simply cease to exist. Used formats may become obsolete and completely unreadable".

8 - "...la conservation répond à deux objectifs principaux:

- elle porte sur des documents servant de pièces justificatives dans le cadre de contrôles administratifs (ex: fiscal ou social) ;

- elle permet la production d'actes juridiques valant preuve en cas de litige. “

Cfr. Eric Caprioli e Gerard Weisz, op. citada.

A este respeito é evidentemente fundamental garantir condições seguras e tecnologicamente eficientes de arquivo dos documentos eletrônicos. "Archiver, c'est conserver un document dont on n'a plus l'utilité immédiate, mais qui peut encore servir, soit à titre informatif, soit à titre de preuve dans l'éventualité d'une contestation, d'un litige devant les tribunaux ou lors d'un contrôle de l'administration", cfr. Valérie Sédallian, «L'archivage de l'acte électronique », Juriscom.net, 8 Juillet 2002 
todo o tempo, pelo menos até ao esgotamento dos prazos prescricionais relativamente às situações por ele comprovadas ${ }^{9}$. Além disso, também há que ter em atenção aspectos relacionados com a própria segurança dos documentos electrónicos e que impõem especiais cuidados no arquivamento e gestão dos documentos electrónicos. É que as exigências de segurança estarão também elas, sujeitas a uma constante e cada vez mais rápida mutação tecnológica. 0 que hoje se tem por seguro, poderá não o ser amanhã. Os requisitos de segurança os documentos electrónicos tendem a evoluir ao ritmo dos próprios desenvolvimentos tecnológicos ${ }^{10}$.

A questão da conservação e arquivo de documentos obviamente não constituía grande problema nas sociedades organizadas em torno de documentos em suporte papel, suporte fiável, durável e imediatamente legível ${ }^{11}$. Mas o caso muda substancialmente de figura ante os novos documentos electrónicos, cujo conteúdo já não é imediatamente perceptível pelo homem, mas somente através da utilização de especiais dispositivos tecnológicos de hardware e software.

Basicamente, a discussão em torno da premente questão da conservação e arquivo dos documentos electrónicos vem apontando algumas possíveis estratégias de ataque do problema. Estas passam essencialmente por uma opção entre duas soluções apontadas como possíveis: a emulação ${ }^{12}$ e a migração.

\section{EMULAÇÃO}

A emulação pressupõe que os dados electrónicos sejam guardados no seu formato original e que os software e hardware necessários à sua leitura sejam mantidos de forma a que a sua ulterior leitura seja sempre possível. Esta solução implica portanto a manutenção dos

\footnotetext{
9 - "Il est impératif de conserver tous les actes au sens large nécessaires à la justification de droits jusqu'à l'expiration des délais prévus pour effectuer une contestation, c'est-à-dire jusqu'à l'expiration du délai de prescription. ", idem.

10 - "Ainsi, à titre d'exemple, selon les experts en cryptographie, le risqué de casser des clés de 128 bits dans les cinq à dix ans n'est pas à negliger. En effet, si pour l'heure, le risque demeure limité il n'est pas écarté. Cela impliquera qu'il faille "resigner" périodiquement le document électronique pour être sûr de son intégrité ou l'assurer par d'autres moyens", cfr. Eric Caprioli e Gerard Weisz, op. citada

11 - "Le papier est considéré comme un support fidèle et durable: la question des modalités de conservation des archives papier n'est pas règlementée et ne fait pas l'objet de controverses juridiques", cfr. Valérie Sédallian, op. citada.

12 - "Emulation...means creating programs for computers of the future so that they can run "old" software and read" old" data. The emulators enable computers to mimic other computers so that today's computers can mimic old ones and computers yet to be invented can mimic today's computers", cfr. Harrison Eiteljorg, "Preservation for the future - with emulation or migration?", CSA Newsletter, Vol. XII, No. 1 Spring, 1999, in http://csanet.org/newsletter/spring99/nls9906.html.
} 
sistemas de software e hardware por longos períodos de tempo ${ }^{13}$. Esta solução teria a enorme vantagem de garantir, a qualquer momento, a leitura de qualquer documento. Por outro lado, a emulação apresenta a enorme vantagem de não implicar (de não necessitar) qualquer tipo de modificação no documento electrónico original ${ }^{14}$. No entanto, há que dizer que ela comporta o grave inconveniente de tornar o arquivo de documentos uma tarefa desmesuradamente pesada para a maior parte das instituições, já que obrigaria as pessoas ou entidades que procedem ao arquivo de documentos a manter, em condições de funcionamento, uma enorme quantidade de equipamentos e de programas de software, de modo a que todos os formatos de software utilizados ao longo dos anos pudessem ser sempre reproduzidos ${ }^{15}$.

\section{MIGRAÇÃO}

A outra possível estratégia para resolver este problema poderá passar já não pela conservação dos formatos, programas e hardware originais, mas antes por um esforço de reconversão de todos os documentos electrónicos para formatos adequados aos novos Standards de software e hardware que vão sendo adoptados no comércio. Assim, os documentos teriam que ser sucessivamente passados para novos formatos, de modo a permitir a sucessiva leitura dos mesmos a partir dos novos equipamentos de hardware e respectivos programas de software ${ }^{16}$. Esta estratégia pressupõe, portanto a chamada "migração" dos documentos electrónicos de um formato para outro.

\footnotetext{
13 . "...the archivist should try to guarantee the usability of electronic data over long periods of time by storing the data in their original format and by making sure that the necessary hardware and software environment enabling the use of these data can always be made available afterwards. This approach is generally called the "emulation " strategy. ", cfr. Jos Dumortier, "E-Government and Digital Preservation", citado, pág. 1.

14 . "...advantage is that the emulation is simply a program running on a machine: using it does not alter the underlying data file in any way", cfr. Harrison Eiteljorg, op. citada.

15 - "Experiments in archiving institutions have demonstrated that this strategy not only requires important efforts and investments but also bears considerable risks", cfr. Jos Dumortier, "E-Government and Digital Preservation", citado, págs.1-2.

16 . "...the archivist may need to convert the document into another format, for instance, in order to keep the document readable on a new hardware and software platform", cfr. Jos Dumortier, "E-Government and Digital Preservation", citado, pág. 2.
} 
Só que também este método encontra grandes dificuldades na sua aplicação, e uma das principais prende-se com a utilização das chamadas assinaturas digitais ${ }^{17}$. É que um dos pressupostos essenciais da utilização deste tipo de assinatura passa precisamente pelo controlo de integridade dos documentos. E a aplicação deste tipo de assinatura leva a que o próprio documento, ou pelo menos a assinatura, só sejam legíveis desde que não haja qualquer alteração do seu conteúdo. 0 que levanta evidentes dificuldades nos casos em que se pretenda migrar os dados electrónicos para novos formatos ou novas plataformas de software. É que, por um lado, a simples modificação de um bit que seja pode tornar um texto (ou uma assinatura) simplesmente ilegível! ${ }^{18}$ Mas por outro lado, também é necessário garantir que a própria assinatura digital pode ser devidamente aplicada, de modo a que o documento seja lido e a origem e integridade do documento verificado, muitos anos depois da criação e assinatura do documento electrónico em causa! ${ }^{19}$

\footnotetext{
17 - questão que se poderá colocar também é a da própria capacidade de conservação da assinatura digital propriamente dita. Cfr. Sofie Van den Eynde, "Digitale Archivering: een juridische stand van zaken vanuit Belgisch perspectief. Deel 1", citado, pág. 83: "De "houdbaarheid" van een digitale handtekening is ook niet onbeperkt. Net zoals de software waarmee een document werd aangemaakt, zullen ook de cryptografische technologieën verouderen waardoor de digitale handtekening niet meer leesbaar is. Bijgevolg zal ere en bepaald procédé moeten toegepast worden op de digitale handtekening, zoals migratie of emulatie, om ze leesbaar te houden" (A "capacidade de conservação" de uma assinatura digital também não é ilimitada. As tecnologias criptográficas também se tornarão antiquadas, tal como o software usado na elaboração de um documento, pelo que a assinatura digital deixará de ser legível. Consequentemente, deverá ser aplicado um determinado procedimento à assinatura digital, como a migração e a emulação, para que esta se mantenha legível).

18 - questão esta que vem colocando algum cepticismo em torno da própria utilização das assinaturas digitais: "Skepticism appeared as soon as it became clear that, when using digital signatures, control of the integrity is only possible if the electronic data remain completely unchanged at the bit-level. This raises a problem when archivists want to migrate electronic data to new formats or software platforms in order to keep them accessible and legible. Some people have immediately concluded that digital signatures are therefore not useful and hence not relevant for archival purposes. ", cfr. Jos Dumortier, "E-Government and Digital Preservation", citado, pág. 5.

19 - parece evidente que, após uma migração, se revestirá da maior importância a realização de cópias de segurança. Cfr. Sofie Van den Eynde, "Wat archiveren en hoe? Op zoek naar de rol van PKI voor digitale archieven" (O que arquivar e como? À procura da função da Infraestrutura de Chaves Públicas para arquivos digitais), http://www.antwerpen.be/david/website/teksten/Rapporten/Rapport6.pdf ponto B.2.3: "Maken van veiligheidskopieën van gearchiveerd informatiepakket" (A elaboração de cópias de segurança dos pacotes de informação arquivados). Mas, na verdade, o que a Autora na realidade propõe é a elaboração de um segundo exemplar do documento: "Het maken van deze kopie mag in geen geval uitgesteld worden. Beide exemplaren moeten idealiter tegelijkertijd gearchiveerd worden. Bovendien moet erover gewaakt worden dat de veiligheidskopie eveneens het origineel bevat. Het word "veiligheidskopie" is hier dus eigenlijk niet op zijn plaats. Beter is te spreken over"het tweede exemplar". (A elaboração dessa cópia não pode, em caso algum, ser adiada. $O$ ideal é que ambos os exemplares sejam arquivados ao mesmo tempo. Além disso, deve ter-se em atenção que a cópia de segurança deve conter também o original. Portanto, o termo "cópia de segurança" não estará correto. Será melhor falar de um "segundo exemplar".
} 


\section{OUTRAS POSSIBILIDADES A CONSIDERAR}

Alguns autores referem como terceira possibilidade de solução do problema do arquivo de documentos electrónicos ao longo do tempo a hipótese de uma real harmonização de formatos. E aponta-se até a possível utilização do formato de documento XML. Mas há que ter em atenção que muitos formatos atualmente utilizados irão certamente sofrer alterações, evoluções e, mais cedo ou mais tarde, acabarão até por ser substituídos. Não é previsível que os standards de facto, ainda que tenham uma utilização generalizada, possam ser mantidos indefinidamente. Pelo que parece que a verdadeira opção se deverá manter entre as hipóteses acima referidas, emulação e migração ${ }^{20}$.

Outra possibilidade que tem sido sugerida, é a da prestação de um serviço, por um terceiro de confiança, que garanta a continuidade da integridade do documento ${ }^{21}$. 0 que está em causa é a atuação de um terceiro de confiança no momento anterior à migração. Este terceiro de confiança verificaria a assinatura digital constante do documento e a integridade e origem do mesmo, após o que faria a migração mediante a aposição de uma nova assinatura digital (ou a um novo ficheiro electrónico que fosse a cópia fiel do anterior, ou a uma extensão do próprio ficheiro electrónico migrado). Claro que esta solução torna inviável uma leitura direta e uma verificação do documento primitivo e da assinatura digital original. No entanto, permitiria garantir com um elevado nível de segurança ${ }^{22}$ que haveria uma continuidade e uma identidade

20 - "The multiple use of XML and its vendor independent character give XML the status of de facto standard. But it is not very likely that XML will be maintained as a common format forever. It will keep evolving and it is unthinkable that there will never be a better alternative for XML. A canonical form that takes all current and future formats into account is unfortunately still IT science fiction. Many archivists therefore believe that there will always remain a need for migration", cfr. Jos Dumortier, "E-Government and Digital Preservation", citado, pág. 5.

21 -. Aleksej Blazic, citado: "A long term Trusted Archive Service should provide a stable environment for preservation of digital data over long periods of time through a regimen of technical and procedural mechanisms".

22 -- que poderia até ser reforçada pela utilização dos chamados meta-dados:. Cfr. Aleksej Blazic, citado: "Some meta information may be associated with the archive data and may deliver descriptive information of data and associated attributes like digital signatures. Additional meta information may be collected during the archival procedure by the TAS" (Trusted Archive Service) "service. Such example is complementary information to digital signatures (digital certificates, certificate revocation lists, etc. ) associated to archive data. 
entre o conteúdo do novo documento e o original ${ }^{23}$. No entanto, algumas dificuldades permaneceriam, dado que a análise da primitiva assinatura electrónica poderia vir a ser necessária para efeitos de prova em Tribunal $^{24}$, sobretudo no caso de haver um repúdio da autoria da mensagem pelo seu putativo autor ${ }^{25}$.

Mas a intervenção de uma terceira parte de confiança poderia servir à manutenção de um conjunto de aplicativos (hardware, software, sistemas operativos) ou pelo menos a um emulador dessas aplicações de modo a garantir a ulterior leitura dos documentos gerados com base nessas

23 - Esta hipótese levanta várias questões interessantes. A primeira é que colocaria mais uma vez em causa o conceito de original de um documento electrónico. Embora muito discutida a possibilidade de original de um documento electrónico, esta questão veio a encontrar desenvolvimentos com a aplicação das assinaturas digitais. A lei canadiana sobre documentos eletrônicos (Loi canadienne sur la proteccion des renseignements personnels et des documents électroniques) considera que "le document électronique pourra satisfaire à l'exigence d'un original émanant de textes legislatifs designées s'il comporte une signature électronique securisée, ajoutée lors de la production originale de document électronique dans sa forme définitive, qui puisse être utilisée pour établir qu'il n'a pas été modifié", cfr. Eric Dunberry, "La preuve et l'archivage des documents électroniques", Wilson \& Lafleur Itée, Montréal, 2000, pág. 87. Mas é evidente que a migração de um documento electrónico pressupõe necessariamente a inviabilização da leitura desse original e a invalidação da primitiva assinatura electrónica. Pelo que tudo se passará como se o documento original tivesse sido destruído e passássemos a dispôr apenas de uma cópia "autenticada" do mesmo. "In this situation, although you would no longer be able to verify the old digital signature directly, you should nonetheless be able to demonstrate continuity of record integrity by verifying the newly digitally signed migrated electronic record and explanatory statement", cfr. Jos Dumortier, "EGovernment and Digital Preservation", citado, pág. 6.

24 .- Importante será sempre a utilização dos chamados selos temporais ou "Time Stamps". Cfr. Aleksej Blazic, citado: "Generally recognized integrity and time existence echanism are the time-stamps techiques as defines in Time-Stamp Protocol Service. These time stamps, issued by Time Stamping Authorities, are signed confirmations that data existed at a certain time. As time stamps are actually digital signatures with a time component delivered by a trusted third party, like Trusted Time Authority (ITA) they suffer from the same time limit restriction as digital signatures". O Regulamento (UE) $\mathrm{n}^{\circ}$ 910/2014 do Parlamento Europeu e do Conselho de 23 de Julho de 2014 relativo à identificação eletrônica e aos serviços de confiança para as transações eletrônicas no mercado interno e que revoga a Diretiva 1999/93/CE, no artigo $3^{\circ} \mathrm{n}^{\circ} 32$ expressamente refere os selos temporais como: "os dados em formato eletrônico que vinculam outros dados em formato eletrônico a uma hora específica, criando uma outra prova de que esses dados existiam nesse momento". E o artigo $41^{\circ}$ do referido Regulamento aborda os efeitos legais dos selos temporais, expressamente prevendo no seu $n^{\circ} 1$ que "Não podem ser negados efeitos legais nem admissibilidade enquanto prova em processo judicial a um selo temporal pelo simples facto de se apresentar em formato eletrônico ...".

25 - "From a legal point of view, in order for signed documents to keep their value over time, it could often be important that the original electronic signature remains present. Signatures could be needed for non-repudiation purposes in an evidential context, for example. “, cfr. Jos Dumortier, "E-Government and Digital Preservation”, citado, pág 6. 0 mesmo autor refere mais adiante que esta questão poderá até parecer estranha em termos históricos, dado que é a primeira vez que se estabelece uma tão nítida separação entre assinatura e documento: "Never before in the history of written communication a signatory has had to worry about how the signature will be linked to the document that he is signing. When using electronic signatures, this becomes now a very relevant issue", cfr. Jos Dumortier, "EGovernment and Digital Preservation", citado, pág 7. 
aplicações $^{26}$. A manutenção de um enorme conjunto de aplicações antigas comportará um enorme custo, só ao alcance de quem, com tais aplicações, possa e pretenda prestar serviços remunerados de certificação ${ }^{27}$. Será possivelmente um negócio a desenvolver na futura sociedade da informação.

No entanto, é sobretudo na hipótese de se optar pela migração que se teria que encarar como absolutamente imprescindível a intervenção de uma entidade terceira de confiança que assegure que o documento que aparece no final da cadeia de documentos migrados é idêntico em conteúdo ao original entretanto perdido e tem origem no "autenticado" autor do documento original $^{28}$. Alguns autores apontam a imprescindibilidade de se garantir, através de serviços de arquivo prestados por terceiros de confiança, o arquivamento da representação binária original do documento ${ }^{29}$. No entanto, também esta possibilidade não está isenta de dificuldades, havendo talvez que considerar aqui a possibilidade de utilização de formatos abertos, que possam ser compreendidos e cujo código fonte possa ser acessado mesmo quando os respectivos formatos deixarem de ser utilizados ${ }^{30}$.

\section{CONCLUSÃO}

A Diretiva Europeia sobre assinaturas electrónicas referia a prestação de serviços de certificação de assinaturas digitais mas não a prestação de serviços de certificação arquivística.

26 - cfr. Jos Dumortier, "E-Government and Digital Preservation", citado, pág 8.

27 - "If one opts for a solution based on emulation, the trusted third party is even more essential. The costs and expertise required for this solution, requires that the task of archiving digital data will be appointed to an independent third party", cfr. Jos Dumortier, "E-Government and Digital Preservation", citado, págs 8-9.

28 . "In the context of preservation based on migration the trusted third party is needed to keep track of the migration process and to make sure that the resulting document at the end of the migration chain keeps being trusted" cfr. Jos Dumortier, "E-Government and Digital Preservation", citado, págs 8-9.

29 - Cfr. Jos Dumortier e Sofie Van den Eynde, "Electronic Signatures and Trusted Archival Services", citado, pág. 7: "The only effective solution in our view for the problem of signature durability, is the archival of the original binary representation of the document. This solution was proposed by the European Electronic Signature Standardization Initiative (EESSI) in the study report "Trusted Archival Services" “.

30 - "To achieve this goal in the best possible way, the TAS "(Serviços de Arquivo de Confiança - Trusted Archival Services)" must only accept documents in a format that can still be understood when the format will no longer be in use. Only open file formats that are vendor-independent qualify for long term archival. Indeed, open file formats, such as XML are being so clearly delineated that the content of such document should be legible even fifty years from now. For today's undocumented, proprietary formats on the contrary (such as Microsoft's Word), it is likely that it won't even be possible to build emulators since no one besides the vendor has an insight in the syntax of these formats", Cfr. Jos Dumortier e Sofie Van den Eynde, "Electronic Signatures and Trusted Archival Services", citado, pág. 7. 
O mesmo se passa com o novo Regulamento Europeu relativo à identificação eletrônica que refere no seu artigo $46^{\circ}$ que “Não podem ser negados efeitos legais nem admissibilidade enquanto prova em processo judicial a um documento eletrônico pelo simples facto de se apresentar em formato eletrônico", mas sem qualquer referência à necessidade de serviços de certificação arquivística . Estranhamente também, a generalidade das leis europeias referentes aos documentos electrónicos preocupam-se com a questão do valor de prova dos documentos electrónicos mas já não com a questão das formas e modalidades de arquivo electrónico e muito menos com a questão crucial da prestação de serviços de arquivo electrónico e de certificação de arquivo electrónico . Ora, é evidente que a própria validade jurídica do documento electrónico há-de estar indissociavelmente ligada aos aspectos aparentemente mais técnicos de conservação dos ficheiros. Na realidade, as implicações jurídicas são evidentes, já que só a permanência, acessibilidade e integridade do documento electrónico podem garantir razoavelmente a segurança jurídica e o valor de prova do documento electrónico . E a própria necessidade de assegurar que as provas estejam disponíveis, eventualmente até para utilização em Tribunal, levam a que esta questão deva ser encarada como um dos aspectos fulcrais a necessitar de trabalho urgente, de cooperação multidisciplinar, entre juristas e informáticos, e de subsequente regulação pelo direito, de modo a tornar o comércio electrónico e a contratação electrónica em geral totalmente viáveis a médio e a longo prazo. Ao fim e ao cabo, são também a confiança das pessoas nos meios electrónicos e a própria segurança das transações que estão em causa.

\section{REFERÊNCIAS}

Sofie Van den Eynde, "Digitale Archivering: een juridische stand van zaken vanuit Belgisch perspectief. Deel 1" (Arquivamento Digital: a situação jurídica atual a partir da perspective belga. Parte 1), Digitale Archivering in Vlaamse Instellingen en Diensten - DAVID, Faculteit Rechtsgeleerheid, Interdisciplinair Centrum voor Recht en Informatica, Leuven 2001.

Jos Dumortier, "E-Government and Digital Preservation", in http://www.unizar.es/derecho/fyd/lefis/documentos/Albareccin_JosDumortier.pdf

Jos Dumortier, "E-Government and Digital Preservation", citada, pag 1. Cfr. ainda Jos Dumortier e Sofie Van den Eynde;"Electronic Signatures and Trusted Archival Services", in http://www.law.kuleuven.ac.be/icri/publications/172DLM202.pdf?where

Sofie Van den Eynde, "Digitale Archivering: een juridische stand van zaken vanuit Belgische perspectief. Deel 1". 